\title{
Estimación de la edad de guanaco (Lama guanicoe) a partir de los dientes incisivos: un aporte metodológico para la construcción de perfiles de mortalidad
}

RMA

Arqueología

\author{
Age estimation of guanaco (Lama guanicoe) based \\ on incisor teeth: a methodological contribution for \\ constructing mortality profiles
}

\author{
Cristian A. Kaufmann*, María Clara Álvarez** y S. Iván Pérez***
}

\begin{abstract}
Resumen
El guanaco fue el principal recurso para los cazadores-recolectores que habitaron las regiones pampeana y patagónica de Argentina. Este ungulado jugó un rol fundamental en las esferas económica, social e ideológica de estas sociedades durante el Holoceno. En este sentido, comprender la relación entre las poblaciones de humanos y guanacos en el pasado es crucial para la zooarquelogía sudamericana. El objetivo de este trabajo es presentar una metodología que permite estimar la edad relativa de los camélidos a partir del análisis de los dientes incisivos. La muestra se compone de incisivos de 91 guanacos modernos, asignados a diferentes clases de edad. Este análisis incluyó variables cualitativas, tales como el estado de la raíz y de la dentina, así como cuantitativas, como la altura de la corona. Los resultados indican que la combinación de estas variables es un indicador útil para la estimación de la edad en incisivos aislados de guanaco, los cuales son frecuentemente hallados en el registro faunístico.
\end{abstract}

Palabras clave: Guanaco; Dientes incisivos; Estimación de la edad; Perfil de mortalidad.

\begin{abstract}
The guanaco was the main resource for hunter-gatherers that inhabited the Pampean and Patagonian regions of Argentina. This ungulate played a fundamental role in the economic, social, and ideological spheres of these societies during the Holocene. In this sense, understanding the relationship between human and guanaco populations in the past is crucial to South American zooarchaeology. The aim of this paper is to present a methodology that allows estimating relative ages of guanaco throughout the analysis of the incisor teeth. The sample is composed of incisors from 91 modern guanacos assigned to different age-classes. This analysis included qualitative variables, such as the root and dentine state, and quantitative ones, such as the crown height. The results show that the combination of these variables is a useful indicator for estimating the age of guanacos in isolated incisors, which are usually found in the faunal record.
\end{abstract}

Keywords: Guanaco; Incisor teeth; Age estimation; Mortality profile.

Los perfiles de mortalidad en sitios arqueológicos derivan de los productos acumulativos de la selección de edad por parte de los predadores (Stiner 1990) y su reconstrucción brinda importante información acerca de las estrategias de subsistencia de las sociedades humanas en el pasado (Driver y Maxwell 2013; Klein 1982). Como han demostrado distintos estudios, la edad es un aspecto importante que condiciona la calidad de la carne, médula, órganos internos y piel, así como la vulnerabilidad y disponibilidad de los animales (Speth 1983; Steele 2002). Es así que a través de los perfiles de mortalidad se pueden conocer las estrategias de caza empleadas por sociedades del pasado y su variación a través del tiempo o, en contextos de domesticación, se pueden inferir las formas de manejo de los rebaños (Greenfield 2005; Payne 1973).

La edad de muerte de un individuo puede ser estimada a partir de métodos que emplean información del proceso de desarrollo ontogénico para diferentes especies de mamíferos (Ruscillo 2006). En zooarqueología, dichos métodos han sido aplicados y refinados para estimar los perfiles de mortalidad de los conjuntos faunísticos (Amorosi 1989; Wilson et al. 1982). Las técnicas frecuentemente utilizadas en la estimación de la edad 
de muerte en especímenes arqueológicos se basan principalmente en la fusión epifisaria y en la erupción combinada con el desgaste dentario (Davis 1987; Klein y Cruz-Uribe 1984; Mengoni Goñalons 1999; O'Connor 2000; Reitz y Wing 1999). La erupción y el desgaste dentario tienen la ventaja de que permiten monitorear la edad de los animales a lo largo de toda la vida, además de que no son una técnica destructiva y no requieren de un equipamiento especializado para su estimación. La mayoría de los investigadores ha registrado la atrición dentaria utilizando códigos de desgaste (Grant 1982; Koike y Ohtaishi 1980; Payne 1973, 1987), en tanto que otros han medido la altura de la corona del diente (Klein et al. 1981; Steele 2002). Para este último caso existen distintos antecedentes, que incluyen principalmente estudios desarrollados sobre bóvidos y équidos (Klein et al. 1981; Klein y Cruz-Uribe 1984; Levine 1983; Spinage 1973; Steele 2002; entre otros). Por último, se han efectuado técnicas microscópicas, como cortes delgados para el análisis del cemento en dientes incisivos y caninos de guanaco (Aldenderfer 1994; Gourichon y Parmigiani 2016; Raedecke 1979).

El guanaco (Lama guanicoe Müller 1776) es el mayor artiodáctilo silvestre sudamericano viviente. Su distribución abarca desde los $8^{\circ}$ sur, en Perú, hasta casi los $55^{\circ}$ sur, en la Isla de Tierra del Fuego (Puig et al. 1997). Este camélido presenta escaso dimorfismo sexual y su peso promedio varía entre 88 y 120 kg (Larrieu et al. 1982; Raedeke 1979). Las hembras alcanzan la edad reproductiva entre los 15 y los 20 meses y el período de gestación dura alrededor de 345 días (Fritz 1985; Raedeke 1979). Los guanacos tienen una expectativa de vida máxima de unos 14 años (Puig y Monge 1983). Son considerados herbívoros generalistas, que se alimentan de una amplia variedad de forraje. Su dieta varía estacionalmente en función de la productividad de su hábitat (Puig et al. 1997). Este animal constituyó una de las principales presas para los cazadores-recolectores del cono sur de Sudamérica, en particular en las regiones de Pampa y Patagonia, durante el Pleistoceno final y todo el Holoceno (Martínez y Gutiérrez 2004; Mengoni Goñalons 1999; Politis 2002; entre otros).

En años recientes, se han desarrollado y perfeccionado los estudios que permiten estimar la edad de muerte de guanacos. Para esto, tradicionalmente se utilizaron secuencias de desarrollo y desgaste dentario (De Lamo 1990; Mengoni Goñalons 2013; Oporto et al. 1979; Puig y Monge 1983; Raedeke 1979). Sin embargo, la altura de la corona es un aspecto que aún no ha sido explorado en la estimación de la edad de este camélido.

En esta publicación se describe de manera cualitativa y métrica la variación de los incisivos a lo largo de la vida de los guanacos. En los sitios arqueológicos de Pampa y Patagonia resulta habitual encontrar estos dientes. Si bien pueden presentarse en posición anatómica, son piezas que se desprenden fácilmente de sus alveolos y suelen hallarse sueltas. Considerando los aspectos mencionados, el objetivo general de este trabajo es desarrollar una metodología que permita estimar edades relativas de guanaco a partir de los incisivos aislados presentes en el registro faunístico.

\section{Características de la dentición de Lama guanicoe}

La dentición del guanaco, así como la del resto de los camélidos, es hetorodonta; es decir, las piezas dentales presentan formas y tamaños diferentes en relación con sus funciones. Los dientes que la integran son incisivos, caninos, premolares y molares (Figura 1). Con excepción de los molares, en una primera etapa de la vida las piezas dentales son deciduas, reemplazadas con la edad por dientes permanentes (dentición difiodonta) (Hillson 1986). Existen pequeñas variaciones en la fórmula dentaria establecida por distintos autores para guanaco (De Lamo 1990; Oporto et al. 1979; Puig y Monge 1983; Raedeke 1979). La observación de la muestra de referencia del presente trabajo indica que la fórmula dentaria coincide con la propuesta para guanacos por Oporto et al. (1979) y para alpacas machos por Fernández-Baca (1971). Sin embargo, algunos autores observaron excepciones, como la presencia del tercer premolar superior permanente junto con otros premolares deciduos, así como casos de hembras que carecen de caninos (de Lamo 1990; Oporto et al. 1979) (Figura 1).

Los dientes incisivos están compuestos por la corona y la raíz. La corona anatómica es la parte del diente que está cubierta por esmalte. Por otro lado, la raíz es la porción del diente que se encuentra inserta en el alveolo y está cubierta por cemento. La raíz posee un orificio denominado foramen apical, por el cual entra el paquete vasculonervioso del diente. Por debajo del esmalte (en la corona) y del cemento (en la raíz) se encuentra la dentina,

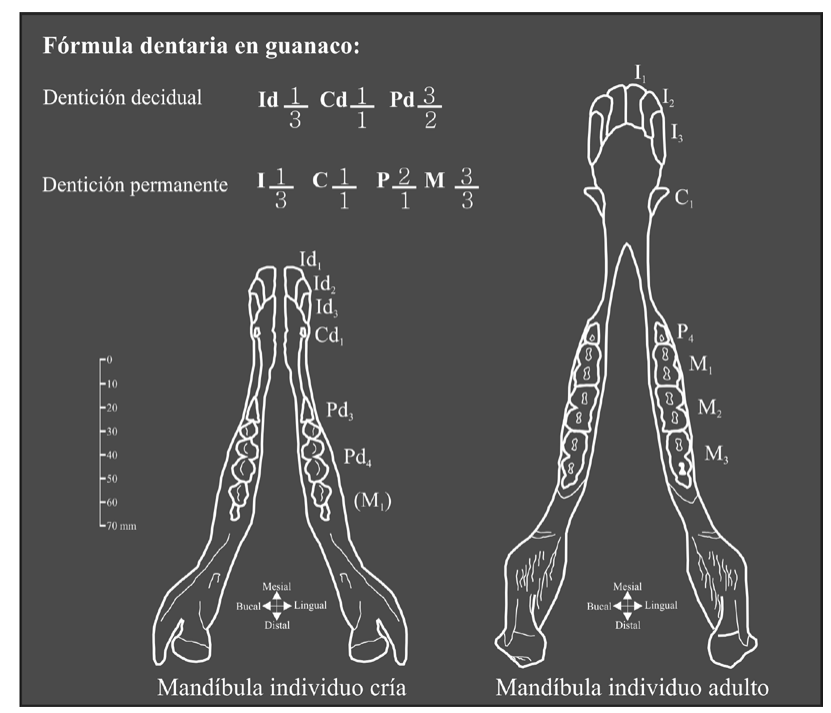

Figura 1. Ilustración esquemática de la dentición mandibular de guanaco cría y adulto.

Figure 1. Schematic illustration of the mandibular dentition from a newborn and an adult guanaco. 
el tejido más voluminoso del diente. La cavidad contenida dentro del diente es la cámara pulpar, estructura que contiene a la pulpa, tejido celular blando vinculado con el suministro de sangre y nervios (Hillson 1986).

El guanaco posee seis incisivos deciduos localizados en el frente de la mandíbula, los cuales son reemplazados por seis incisivos permanentes. Estos dientes tienen superficies en forma de bisel y son utilizados para cortar vegetales contra la encía opuesta, ubicada en el frente de la maxila (Oporto et al. 1979). Los dos incisivos superiores son puntiagudos, adoptan forma de caninos y se hallan separados de los premolares y molares por un diastema. Los caninos inferiores también están divididos por un diastema de los incisivos, premolares y molares. Los premolares y molares son de tipo selenodonte, están formados por cúspides alargadas en forma de pliegues semilunares y poseen infundíbulos y raíces diferenciadas. Hemos observado que los incisivos deciduos poseen diámetros de la raíz más pequeños y coronas más angostas y cortas que las de los permanentes (Figura 2).

No existen trabajos previos que evalúen la altura de la corona en incisivos de guanaco, pero hay referencias acerca de la descripción de ciertos aspectos cualitativos de estos dientes. Algunos antecedentes han establecido secuencias de erupción y desgaste de los incisivos sobre la base del grado de exposición de la dentina y la morfología de la superficie oclusal. En general, los autores coinciden en que al momento del nacimiento los incisivos ya han erupcionado o están próximos a hacerlo (De Lamo 1990; Oporto et al. 1979; Puig et al. 1997; Raedeke 1979). Entre los tres y cinco meses comienza a observarse una fina línea de dentina, debido al desgaste de la cara oclusal. Entre los 20 y 36 meses se produce el cambio de los incisivos deciduos por los permanentes. Estos últimos evidencian un fuerte desgaste luego de los cinco a seis años, momento en que su morfología se torna oval y se observa la cámara pulpar a través de un punto oscuro en la parte central del diente (De Lamo 1990; Oporto et al. 1979; Puig et al. 1997; Raedeke 1979). Por otra parte, Mengoni Goñalons (2013:317) observó que la raíz de los incisivos deciduos se encuentra casi cerrada hacia los ocho a nueve meses de edad y que en los permanentes el cierre se produce casi simultáneamente, ya que los tres incisivos son reemplazados en un corto plazo.

\section{Materiales y Métodos}

La muestra utilizada para llevar a cabo este análisis corresponde a ejemplares modernos procedentes de la localidad de Cinco Chañares, departamento de San Antonio, provincia de Río Negro, Patagonia Argentina (403' $33^{\prime}-40^{\circ} 40^{\prime}$ sur y $65^{\circ} 11^{\prime}-65^{\circ} 32^{\prime}$ oeste). Esta región posee un clima árido, de tipo semi-desértico y templado, fuertemente condicionado por los vientos secos que penetran al continente desde el oeste y el noroeste (Guarido y Mazzitelli 2003). El área se encuentra en la provincia biogeográfica del Monte, la cual ocupa una

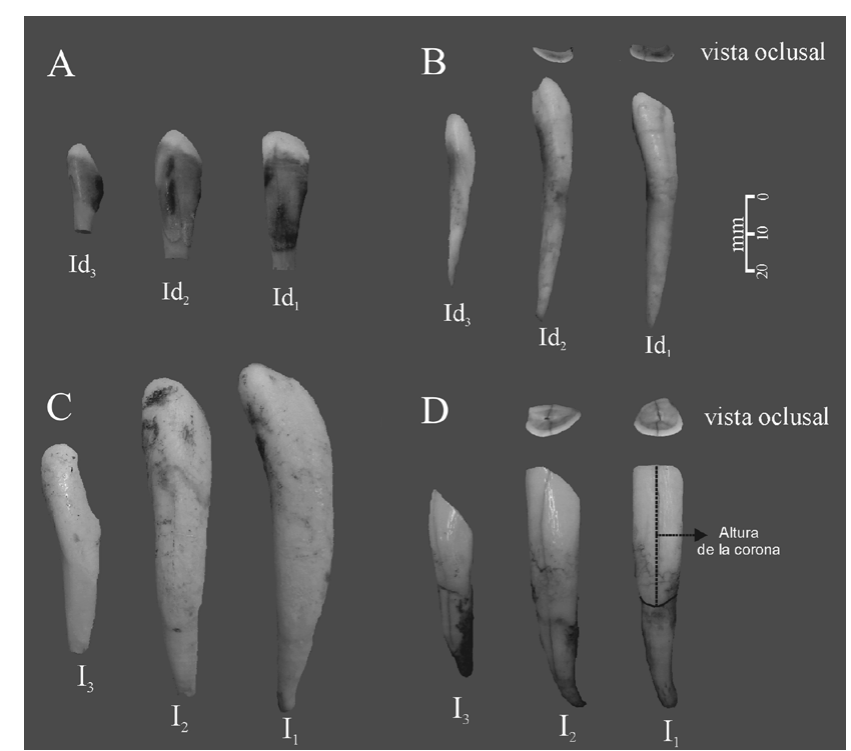

Figura 2. Vista bucal de incisivos deciduos y permanentes y ejemplo de medición de la altura de la corona.

Figure 2. Buccal view of deciduous and permanent incisors, and example of crown height measurement.

gran extensión del territorio argentino (paralelo 27 al 44: desde la provincia de Salta hasta el Nordeste de Chubut; Cabrera y Willink 1980). La localidad de Cinco Chañares se incluye en la subregión Monte Austral (Morello 1958) y limita al norte con la provincia del Espinal y al suroeste con la provincia patagónica.

La muestra estudiada está representada por 91 individuos: tres nonatos (siete a 11 meses de gestación), 23 crías (cero meses a un año), 20 juveniles (uno a dos años), 15 subadultos (dos a tres años), 18 adultos (tres a siete años) y 12 seniles (más de siete años). En una primera instancia, se ordenaron los individuos sobre la base del desarrollo y desgaste que presentaban los dientes mandibulares. Los esqueletos fueron agrupados en distintas categorías etarias teniendo en cuenta las secuencias de edad elaboradas por Oporto et al. (1979) y De Lamo (1990). En algunos guanacos juveniles se conocía la fecha de muerte y por este motivo pudo estimarse la edad, considerando que los nacimientos se producen en una temporada acotada del año (Raedeke 1979:362). El sexo fue determinado para la clase subadulta y las subsiguientes, por medio de la observación de los dientes caninos y la pelvis. De esta manera, se determinó que 10 de los individuos corresponden a machos y 23 a hembras, en tanto que 12 resultaron indeterminados. Para los nonatos, crías y juveniles no se pudo determinar el sexo, dado que los caracteres sexuales se encontraban en vías de desarrollo.

Para cada individuo se relevó la presencia/ausencia de los incisivos deciduos y/o permanentes. Luego se realizó la extracción manual de los dientes de sus alveolos. A partir de esto pudo describirse el estado de la raíz; abierta, casi cerrada o cerrada. En este último caso sólo se observa el foramen apical. Además, se detallaron las características de cada diente y se realizaron ilustraciones 
esquemáticas de la cara oclusal. Se relevó la presencia o ausencia de dentina, observable a partir del desgaste del esmalte. Cuando ésta era visible se realizaron ilustraciones para dar cuenta de la forma que adquiría la superficie oclusal del incisivo. A partir de estos datos se elaboraron categorías de desgaste considerando rangos amplios de edad. Paralelamente, uno de los autores (MCA) tomó las medidas del ancho de la dentina, teniendo en cuenta la distancia entre las paredes internas del esmalte de la cara vestibular y lingual del incisivo. Para esto se utilizó un calibre digital de operación manual Digi Log (precisión 0,01 mm). Finalmente, se midió (MCA) la altura de la corona anatómica de los incisivos en su cara bucal. Dicha medida corresponde a la distancia mínima entre el punto medio de la superficie oclusal y el punto medio del cuello del incisivo (ver Figura 2). Se registraron tres series de observaciones sobre 31 especímenes. Además, se obtuvieron las mismas medidas dentales espaciadas en siete días (MCA). El error de observación fue evaluado empleando una prueba de correlación y un ANOVA de medidas repetidas. Los resultados obtenidos mostraron que este es desechable, dado que las series de observación fueron significativamente similares $(r=0,999, F=0,494$, $\mathrm{p}=0,487)$. Todos los análisis estadísticos aplicados fueron realizados en el programa $R$, versión 3.3.0 (R Core Team 2016).

\section{Resultados}

Los datos para el total de los 91 individuos analizados pueden encontrarse como material suplementario online (Tabla suplementaria 1).

\section{Aspectos cualitativos de la Raíz}

Para los incisivos deciduos se observó que ya se encuentran en desarrollo en el último tercio de gestación. La raíz permanece abierta hasta los seis meses, en el 100\% de los casos. Entre los seis y nueve meses predomina la raíz abierta o casi cerrada, aunque se registra un caso de raíz cerrada. Entre los nueve y 19 meses la raíz está casi cerrada o cerrada. A partir de los 19 meses, en el 100\% de los casos la raíz está cerrada (Tabla 1). A su vez, se observó que a partir del año de edad existen algunos casos de reabsorción de la raíz, vinculados con la erupción de las piezas permanentes.

En el caso de los incisivos permanentes, entre los 30 y 36 meses la raíz se encuentra abierta en todos los casos. Entre los 36 y 48 meses se registran especímenes en los que está abierta, casi cerrada y cerrada. Finalmente, a partir de los 48 meses de edad, la raíz está cerrada en todos los individuos (Tabla 1).

Si se considera el estado de la raíz como criterio para la estimación de la edad podrían determinarse los grupos que se mencionan a continuación. Para los incisivos deciduos, entre los 10 meses de gestación y los nueve meses (raíz abierta) y entre los nueve y 30 meses (raíz cerrada). En
Tabla 1. Estado de la raíz para los incisivos permanentes y deciduos presentes en la muestra.

Table 1. Root state for permanent and deciduous incisors from the sample.

\begin{tabular}{|c|c|c|c|c|c|c|c|c|c|}
\hline & \multicolumn{4}{|c|}{ I1d } & \multicolumn{2}{|c|}{$12 d$} & \multicolumn{3}{|c|}{ I3d } \\
\hline Edad & A & $\mathrm{CC}$ & C & A & $\mathrm{CC}$ & C & A & $\mathrm{CC}$ & C \\
\hline 10-11 MG & 3 & - & - & 3 & - & - & 3 & - & \\
\hline $0-15 \mathrm{D}$ & 3 & - & - & 2 & - & - & 2 & - & - \\
\hline $15 \mathrm{D}-3 \mathrm{M}$ & 3 & - & - & 3 & - & - & 3 & - & - \\
\hline 3-6 M & 7 & - & - & 7 & - & - & 7 & - & - \\
\hline $6-9 \mathrm{M}$ & 2 & 2 & - & 2 & 2 & - & 2 & 1 & 1 \\
\hline $9-12 \mathrm{M}$ & - & 3 & 3 & - & 3 & 3 & - & 1 & 5 \\
\hline $12-19 \mathrm{M}$ & - & 5 & 9 & - & 5 & 9 & - & 3 & 10 \\
\hline $19-24 \mathrm{M}$ & - & - & 4 & - & 0 & 5 & - & - & 6 \\
\hline \multirow[t]{2}{*}{$24-30 \mathrm{M}$} & - & - & 8 & - & 0 & 10 & - & - & 10 \\
\hline & \multicolumn{3}{|c|}{$11 p$} & \multicolumn{3}{|c|}{$12 p$} & \multicolumn{3}{|c|}{$13 p$} \\
\hline $30-36 \mathrm{M}$ & 4 & - & - & 4 & - & - & 4 & - & - \\
\hline $36-48 \mathrm{M}$ & 1 & 3 & 2 & 2 & 2 & 2 & 3 & 3 & 1 \\
\hline $48-60 \mathrm{M}$ & - & - & 2 & - & - & 2 & - & - & 2 \\
\hline $60-72 \mathrm{M}$ & - & - & 8 & - & - & 8 & - & - & 8 \\
\hline $72-84 \mathrm{M}$ & - & - & 2 & - & - & 2 & - & - & 2 \\
\hline 84-96 M & - & - & 2 & - & - & 2 & - & - & 2 \\
\hline 96-108 M & - & - & 3 & - & - & 3 & - & - & 3 \\
\hline 120-132 M & - & - & 3 & - & - & 3 & - & - & 2 \\
\hline $132-148 \mathrm{M}$ & - & _ & 2 & & - & 2 & - & - & 2 \\
\hline
\end{tabular}

Referencias: $M G=$ meses de gestación, $D=$ días, $M=$ meses, $A=$ abierta, $\mathrm{CC}=$ casi cerrada, $\mathrm{C}=$ cerrada.

el caso de los incisivos permanentes, entre los 30 y 48 meses (raíz abierta) y más de 48 meses (raíz cerrada). En la muestra también se identificaron algunos casos correspondientes al momento en el cual la raíz comienza a cerrarse. Con fines operativos, estos especímenes en los cuales la raíz estaba casi cerrada fueron incorporados al grupo de raíz cerrada.

\section{Aspectos cualitativos de la Dentina}

En el incisivo 1 deciduo la dentina comienza a observarse a partir de los tres meses y en el incisivo 2 a partir de los nueve meses. Para los incisivos permanentes, la dentina se expone a partir de los 30 meses en el incisivo 1 y de los 36 meses en el 2. En el caso del incisivo 3, debido a la variabilidad en la exposición de dentina, no se definieron categorías.

Una vez que comienza a observarse la dentina, empieza a cambiar la forma del plano oclusal, el cual se ve más ancho, ya que a partir del desgaste la corona pierde altura. La morfología pasa de una fina línea a una forma triangular y de ésta a una más ovoide. Cuando el guanaco es adulto comienza también a hacerse visible la cavidad pulpar. Además, cuando el desgaste es muy avanzado, el esmalte que rodea a la dentina comienza a desaparecer en la cara mesial, en primera instancia, y luego en la cara lingual, permaneciendo sólo en la cara bucal.

Considerando la morfología de la superficie oclusal, se definieron tres categorías para los incisivos 1 y 2, tanto deciduos (d) como permanentes (p). Para los deciduos, la 
Figura 3. Categorías de desgaste de la superficie oclusal de los incisivos deciduos y permanentes.

Figure 3. Dentin wear categories for the occlusal surface in deciduous and permanent incisors.

\begin{tabular}{|c|c|c|c|c|c|}
\hline Edad & $7 \mathrm{mg}-9 \mathrm{~m}$ & $9-19 \mathrm{~m}$ & $19-30 \mathrm{~m}$ & $30-60 \mathrm{~m}$ & $+\operatorname{de} 60 \mathrm{~m}$ \\
\hline $\begin{array}{c}\text { Cat. de desgaste } \\
\text { I1-2d }\end{array}$ & $\mathbf{1 d}$ & $\mathbf{2 d}$ & $\mathbf{3 d}$ & & \\
\hline $\begin{array}{c}\text { Estado de la } \\
\text { superficie oclusal }\end{array}$ & & & $\mathbf{1 p}$ & $\mathbf{2 p}$ & $\mathbf{3 p}$ \\
\hline $\begin{array}{c}\text { Cat. de desgaste } \\
\text { I1-2p }\end{array}$ & & & & & \\
\hline $\begin{array}{c}\text { Estado de la } \\
\text { superficie oclusal }\end{array}$ & & & & & \\
\hline
\end{tabular}

Categoría 1d abarca desde los siete meses de gestación a los nueve meses. En ésta no se observa dentina o bien se trata de una fina línea. En la Categoría 2d, de nueve a 19 meses, la dentina adquiere una forma alargada ondulante. Para la Categoría 3d (19 a 30 meses) las piezas adoptan una forma sub-triangular a ovoide. En cuanto a los incisivos permanentes, en la Categoría 1p (19 a 30 meses) no se observa dentina, ya que toda la cara oclusal está cubierta por esmalte. En la Categoría 2p (30 a 60 meses) se observa una fina línea de dentina que va incrementándose gradualmente, adoptando una forma triangular. En la Categoría $3 p$ la cara oclusal adquiere una forma ovoide y en la mayoría de los casos se observa la cámara pulpar (Figura 3). En el caso de los individuos que tienen entre 19 y 30 meses, suelen encontrarse mandíbulas con incisivos deciduos (Categoría 3d) con avanzado desgaste, junto con permanentes en proceso de erupción (Categoría 1p). Este aspecto es importante para tener en cuenta a la hora de estimar el número mínimo de individuos.

Para el estado de la raíz y la forma de la superficie oclusal se realizaron correlaciones de Spearman considerando la edad conocida y la obtenida a partir del método propuesto. Para el estado de la raíz las correlaciones con la edad conocida fueron altas, siendo de $R_{s}=0,973$ para el incisivo $1, R_{s}=0,976$ para el incisivo 2 y $R_{s}=0,976$ para el incisivo 3. Para el estado de la superficie oclusal las correlaciones tuvieron valores similares, siendo de $R_{s}=0,984$ para el incisivo 1 y de $R_{s}=0,964$ para el incisivo 2 .

\section{Altura de la Corona}

En relación con las variables cuantitativas, se llevaron a cabo regresiones lineales y cuadráticas para determinar la relación entre la altura de la corona y la edad (estimada por el desarrollo y desgaste de los molares). En los gráficos se observa que para los incisivos 1 y 2 deciduos hay una relación débil entre la altura la corona y la edad, con valores de ajuste por debajo de un $\mathrm{R}^{2}=0,70$. Para el caso del incisivo 1 y 2 permanentes el ajuste entre la altura de la corona y la edad es más alto, alrededor del 0,80, siendo un poco mejor para el 1. En el caso del incisivo 3 el ajuste del incisivo deciduo es malo, en tanto que para el permanente es mejor, de alrededor de un $\mathrm{R}^{2}=0,70$ (Figura 4). En archivo suplementario se presentan las fórmulas de ajuste lineal y cuadrático, a partir de las cuales se pueden ingresar las medidas de la altura de la corona para obtener una edad estimada (Archivo suplementario 1).
A medida que aumenta el desgaste de los incisivos, resulta más difícil diferenciar entre los dientes 1 y 2, dado que son muy similares morfológicamente. Por este motivo, se obtuvieron ajustes de regresión lineal y cuadrática para ambas piezas en conjunto. Los resultados indican que el ajuste $\left(R^{2}\right)$ fue de alrededor de 0,80 para los dientes permanentes y de 0,65 para los deciduos. Para los casos de análisis en que no pueden diferenciarse los incisivos 1 del 2 se presentan también las fórmulas del modelo lineal y el cuadrático a partir de ambos dientes para obtener la edad estimada (Archivo suplementario 1). Es importante señalar que aunque los intervalos de la predicción son
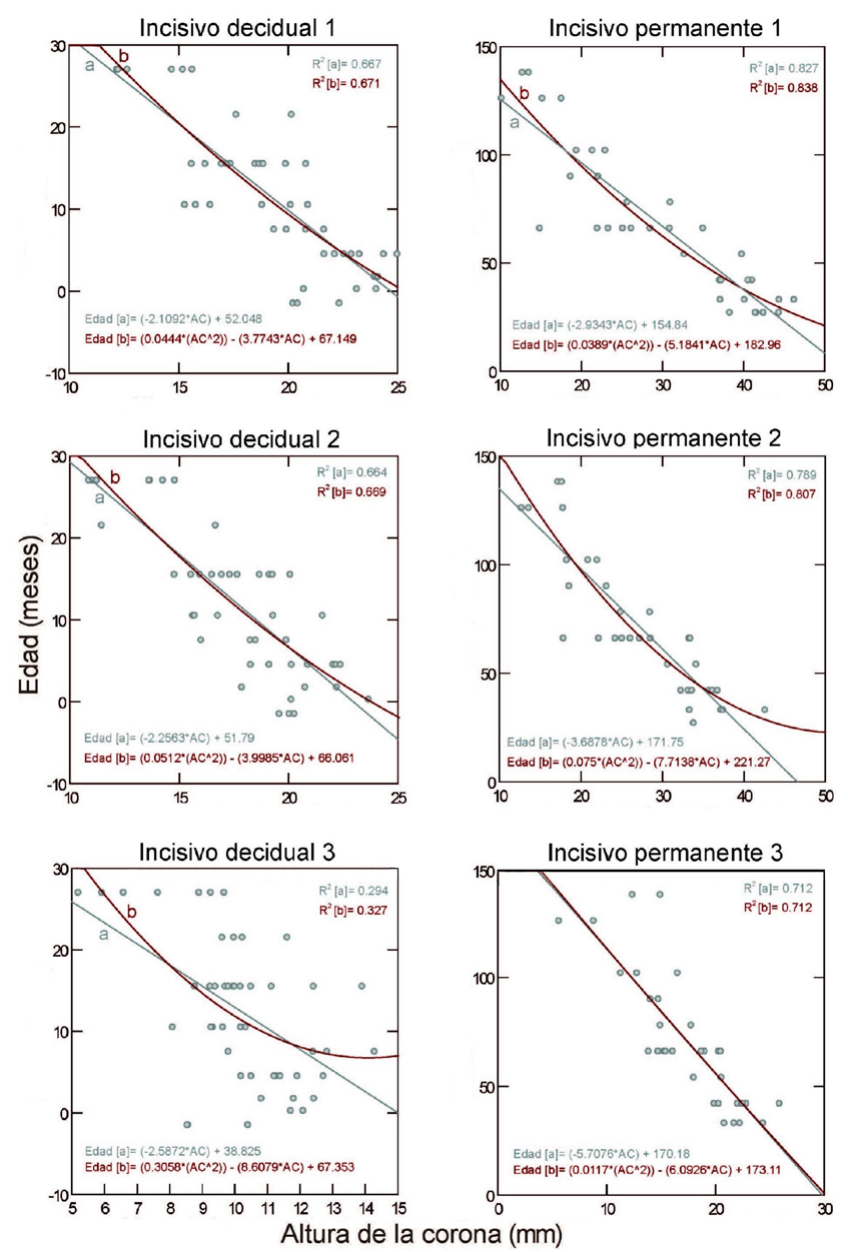

Figura 4. Relación entre la altura de la corona y la edad para los incisivos deciduos y permanentes.

Figure 4. Relation between crown height and age in permanent and deciduous incisors. 
relativamente grandes para todos los dientes estudiados (Figura suplementaria 1), la edad estimada y la original muestran una correlación fuerte para los dientes permanentes (Figura suplementaria 2).

\section{Comentarios Finales}

En este trabajo se desarrolló un método para estimar la edad relativa de guanaco a partir de los incisivos aislados presentes en el registro faunístico. Este combina tres indicadores, dos cualitativos y uno métrico, que pueden emplearse de manera complementaria. Para la aplicación de estos criterios es necesario, en primera instancia, dividir la muestra en incisivos deciduos y permanentes, considerando la morfología externa y el tamaño de cada diente. Esta primera división permite separar dos grandes grupos de edad, juveniles y adultos.

Posteriormente, se emplean los dos criterios cualitativos más específicos, el estado de la raíz y la forma de la superficie oclusal. El primero de estos criterios permite una nueva división de la muestra en los grupos de edad crías y juveniles, para los incisivos deciduos, y adultos jóvenes y adultos reproductivos/seniles, para los incisivos permanentes. El segundo criterio cualitativo, el estado y la forma de la superficie oclusal del incisivo, tiene un mayor grado de especificidad. Los dientes deciduos pueden separarse en crías, juveniles y subadultos. Esta última categoría también está representada por los dientes permanentes de raíz abierta. Asimismo, a partir de estas piezas se puede discriminar la clase de adultos reproductivos de la de adultos seniles. Los análisis de correlaciones de Spearman considerando la edad conocida y la obtenida a partir del método propuesto mostraron que ambos criterios cualitativos resultaron muy buenos para la estimación de la edad en categorías amplias.

El criterio métrico permite, a diferencia de los cualitativos, la aplicación de una fórmula para obtener una edad numérica. El mismo resultó más efectivo para los incisivos permanentes, es decir, subadultos, adultos reproductivos y adultos seniles, y puede ser empleado como un criterio independiente de los cualitativos. En relación con esto, puede realizarse una estimación de la edad medida en meses, incluyendo intervalos de confianza de la estimación. Las fórmulas para la altura de la corona (tanto la lineal como la cuadrática), pueden ser aplicadas con un error aceptable para determinar la edad de muerte a partir de un espécimen. Este error fue de \pm tres meses cuando se emplearon los dientes deciduos y de \pm cinco a ocho meses cuando se utilizaron los permanentes.

En trabajos previos, se ha utilizado para la estimación de la edad de muerte en guanacos, la fusión epifisaria (Herrera 1988; Kaufmann 2009; Mengoni Goñalons 1999) y la secuencia de erupción, desarrollo y desgaste para molares (Kaufmann 2009). El análisis de los incisivos es otro indicador que provee especificidad y brinda información relevante que se complementa con la de los demás elementos o sirve como estimación viable en su ausencia. La existencia de múltiples indicadores de la edad, en combinación con la determinación del sexo (Kaufmann 2009; Kaufmann y L'Heureux 2009; Kaufmann et al. 2013), permite reconstruir perfiles de mortalidad a partir de elementos óseos que poseen diferente densidad mineral ósea (Elkin 1995; Stahl 1999), utilidad económica y potencial para su consumo diferido (Borrero 1990; De Nigris y Mengoni Goñalons 2005). Las distintas unidades anatómicas podrían tener mayores o menores chances de ser transportadas por los grupos humanos desde los sitios de matanza hacia sus campamentos residenciales (Binford 1978), así como de sobrevivir ante procesos naturales como la meteorización (González et al. 2012), el transporte fluvial (Kaufmann et al. 2011) y la acción de carnívoros (Munson y Garniewicz 2003; Kaufmann et al. 2016), entre otros. Es por esto que es importante considerar distintos indicadores de la edad de manera simultánea, aspecto que dará mayor robustez a las interpretaciones que se realicen sobre la variabilidad de las estrategias de caza de los grupos humanos en el pasado.

Todos los indicadores de sexo o edad son específicos de cada población. El desgaste, por su etiología, es uno de los que más varía entre poblaciones. Sin embargo, si las condiciones de las poblaciones fueran similares, estas fórmulas podrían usarse (críticamente) en otras poblaciones. Es necesario considerar que la cantidad de partículas abrasivas que forma parte de los alimentos (fitolitos) y que está presente en el ambiente, podría generar un mayor o menor desgaste. Sin embargo, dado que la oclusión se conserva, la morfología se mantiene en la evolución del desgaste (Fortelius y Solounias 2000; Kaiser et al. 2013). Esto implica que si bien podría diferir el tiempo implicado para alcanzar cada una de las categorías en guanaco, la secuencia de los estadíos sería la misma. Por este motivo, al momento de trabajar con una muestra faunística es importante aplicar los tres criterios presentados en este trabajo. La combinación de los criterios métricos con los cualitativos podría garantizar una mayor objetividad en la determinación de la edad de muerte. En este sentido, los criterios cualitativos son tan importantes como los métricos al momento de estimar la edad de muerte a partir de un espécimen. Por su parte, algunas variables, como la altura de la corona, están más influenciadas por el ambiente que otras, como el estado de la raíz. En conclusión, se sugiere emplear un enfoque de múltiples indicadores para la construcción de los perfiles de mortalidad, incorporando la mayor cantidad de variables posibles.

\section{Agradecimientos}

Este trabajo se desarrolló en el marco del INCUAPACONICET (Facultad de Ciencias Sociales, Universidad Nacional del Centro de la Provincia de Buenos Aires). Queremos agradecer al Museo de las Ciencias (Olavarría), por brindarnos la infraestructura para analizar y almacenar las colecciones de referencia. A Lorena L'Heureux, por sus valiosos comentarios sobre el desarrollo de la metodología 
propuesta. Finalmente, a dos evaluadores anónimos, por sus sugerencias y modificaciones a la versión original de este artículo.

\section{Bibliografía}

Aldenderfer, M. 1994. Dental microstructure analysis as a means of age/seasonality estimation in camelids. D. Elkin, C. Madero, G. Mengoni Gonalons, D. Olivera, M.d.C. Reigadas y H. Yacobaccio editores, Zooarqueología de camélidos. Perspectivas teóricas y metodológicas vol. 1, pp. 9-21. Grupo de Zooarqueología de Camélidos, Buenos Aires.

Amorosi, T. 1989. A postcranial guide to domestic neonatal and juvenile mammals. BAR International Series British Archaeological Reports, Oxford.

Binford, L. 1978. Nunamiut ethnoarchaeology. Academic Press, New York.

Borrero, L. A. 1990. Fuego-Patagonia bone assemblage and the problem of communal guanaco hunting. L. Davis y B. Reeves editores, Hunters of the Recent Past, pp. 373-399. Londres, Unwin Hyman.

Cabrera, A. L. y A. W. Willink. 1980. Biogeografía de América Latina. Serie de Biología. Monografía Nro. 13. Secretaría General de la Organización de los Estados Americanos. Programa Regional de Desarrollo Científico y Tecnológico, Washington D.C.

Davis, S. J. 1987. The archaeology of animals. Yale University Press, New Haven.

De Lamo, D. A. 1990. Determinación de edad en guanacos (Lama guanicoe Müller) por análisis del desarrollo y desgaste dentario. Veterinaria Argentina 7 (69): 621-625.

De Nigris, M. y G. Mengoni Goñalons. 2005. The guanaco as a source of meat and fat in the Southern Andes. J. Mulville y A. Outram editores, The Zooarchaeology of the Fats, Oils, Milk and Dairying, pp. 160-166. Oxbow Books, Oxford.

Driver, J. C. y D. Maxwell. 2013. Bison death assemblages and the interpretation of human hunting behavior. Quaternary International 297: 100-109.

Elkin, D. 1995. Volume density of south american camelids skeletal parts. International Journal of Osteoarchaeology 5: 29-37.

Grant, A. 1982. The use of tooth wear as a guide to the age of domestic ungulates, B. Wilson, C. Grigson y S. Payne editores, Ageing and sexing animal bones from archaeological sites, pp. 91-108. BAR International Series British Archaeological Reports, Oxford.

Fernández-Baca, S. 1971. La alpaca: reproducción y crianza. Boletín de Divulgación 9 (7). Instituto Veterinario de Investigaciones Tropicales y de Altura, Lima, Perú.

Fortelius, M. y N. Solounias. 2000. Functional characterization of ungulate molars using the abrasion-attrition wear gradient:
A new method for reconstructing paleodiets American Museum Novitates 3301:1-36.

Fritz, M. A. 1985. "Population dynamics and preliminary estimates of the harvestability of the Patagonia guanaco". Tesis de Maestría Inédita, lowa State University, Ames.

González, M. E., M. C. Álvarez, A. Massigoge, M. A. Gutiérrez y C. A. Kaufmann. 2012. Bone differential survivorship and ontogenetic development in guanaco (Lama guanicoe). International Journal of Osteoarchaeology 22: 523-536.

Gourichon, L. y V. Parmigiani. 2016. Preliminary analysis of dental cementum of Lama guanicoe for the estimation of age and season at death: Studies of modern specimens and further archaeological applications. Journal of Archaeological Science: Reports 6: 856-861.

Greenfield, H. J. 2005. A reconsideration of the secondary products revolution in southeastern Europe: on the origins and use of domestic animal milk, wool, and traction in the central Balkans, J. Mulville y A. Outram editores, The Zooarchaeology of Fats, Oils, Milk and Dairying, pp. 14-31. Oxbow Books, Oxford.

Guarido, J. C. y M. Mazzitelli. 2003. Geografía. El Bajo del Gualicho, R. F. Masera y J. C. Guarido editores, Bajo del Gualicho: una planicie patagónica bajo el nivel del mar. Realidad y leyenda, pp. 25-57. Secretaria de Estado de Acción Social de Río Negro. Carmen de Patagones, Río Negro.

Herrera, O. 1988. Los camélidos y sus indicadores óseos de estacionalidad: apuntes para la discusión, A. Haber y N. Ratto editores, De procesos, contextos y otros huesos, pp. 101-110. Facultad de Filosofía y Letras, UBA. Buenos Aires.

Hillson, S. 1986. Teeth. Cambridge University Press, Cambridge.

Kaiser, T. M., D. W. H. Müller, M. Fortelius, E. Schulz, D. C. y M. Clauss. 2013. Hypsodonty and tooth facet development in relation to diet and habitat in herbivorous ungulates: implications for understanding tooth wear. Mammal Review 43 (1): 34-46.

Kaufmann, C. A. 2009. Estructura de edad y sexo en Lama guanicoe (guanaco). Estudios actualísticos y arqueológicos en Pampa y Patagonia. Sociedad Argentina de Antropología, Buenos Aires, Argentina.

Kaufmann, C. A. y G. L. L'Heureux. 2009. El dimorfismo sexual en guanacos (Lama guanicoe). Una evaluación osteométrica de elementos poscraneales. Revista del Museo de Antropología 2:181-198.

Kaufmann, C. A., M. A. Gutiérrez, M. C. Álvarez, M. E. González y A. Massigoge. 2011. Fluvial dispersal potential of guanaco bones (Lama guanicoe) under controlled experimental conditions: the influence of age classes to the hydrodinamic behavior. Journal of Archaeological Science 38 (2): 334-344.

Kaufmann, C.A., M. C. Álvarez, L. G. L’Heureux y M. A. Gutiérrez. 2013. Dimorfismo sexual en la pelvis de Lama guanicoe (Artiodactyla, Camelidae): un caso de aplicación en el sitio Paso Otero 1, Buenos Aires, Argentina. Mastozoología Neotropical 20 (1): 47-59. 
Kaufmann, C. A., D. J. Rafuse, M. E. González, M. C. Álvarez, A. Massigoge, N. A. Scheifler y M. A. Gutiérrez. 2016. Carcass utilization and bone modifications on guanaco killed by puma in northern Patagonia, Argentina. Quaternary International. En Prensa. doi:10.1016/j.quaint.2016.03.003.

Klein, R. G. 1982. Patterns of ungulate mortality and ungulate mortality profiles from Langebaanweg (Early Pliocene) and Elandsfontein (Middle Pleistocene), south-western Cape Province, South Africa. Annals of the South African Museum 20: 49-94.

Klein, R. G., C. Wolf, L. G. Freeman y K. Allwarden. 1981. The use of dental crown heights for constructing age profiles of red deer and similar species in archaeological samples. Journal of Archaeological Science 8: 1-31.

Klein, R. G. y K. Cruz-Uribe. 1984. The analysis of animal bones from archeological sites. University of Chicago Press, Chicago, Illinois.

Koike, H. y N. Ohtaishi. 1985. Prehistoric hunting pressure estimated by the age composition of excavated sika deer (Cervus nippon) using the annual layer of tooth cement. Journal of Archaeological Science 12: 443-456.

Larrieu, E., R. Bigatti, C. Eddi, E. Bonazzi, E. Gomez, R. Niec y N. Oporto. 1982. Contribución al estudio del estudio del parasitismo gastrointestinal en guanacos (Lama guanicoe) y llamas (Lama glama). Gaceta Veterinaria 54: 958-960.

Levine, M. A. 1983. Mortality models and the interpretation of horse population structure, G. Bailey editor, Hunter-Gatherer Economy in Prehistory: A European Perspective, pp. 23-46. Cambridge University Press, Cambridge.

Martínez, G. y M. A. Gutiérrez. 2004. Tendencias en la explotación humana de la fauna durante el Pleistoceno final y Holoceno en la Región Pampeana (Argentina), G. L. Mengoni Goñalons editor, Zooarchaeology of South America, pp. 81-98. BAR International Series 1298, Oxford.

Mengoni Goñalons, G. L. 1999. Cazadores de guanaco de la estepa patagónica. Sociedad Argentina de Antropología. Buenos Aires.

Mengoni Goñalons, G. L. 2013. El aprovechamiento de la fauna en sociedades complejas: aspectos metodológicos y su aplicación en diferentes contextos arqueológicos del NOA, V. Williams y M. B. Cremonte editores, Al borde del imperio. Paisajes sociales, materialidad y memoria en áreas periféricas del Noroeste argentino, pp. 311-396. Sociedad Argentina de Antropología, Buenos Aires.

Morello, J. 1958. La provincia fitogeográfica del Monte. Opera Lilloana 2: 1-155.

Munson, P. J. y R. C. Garniewicz. 2003. Age-mediated survivorship of ungulate mandibles and teeth in canid-ravaged faunal assemblages. Journal of Archaeological Science 30: 405-416.

O' Connor, T. 2000. The archaeology of animal bones. Texas A \& M University Press, College Station, Sutton, Stroud.
Oporto, N., R. Bigatti y E. Larrieu. 1979. Determinación de edades en guanaco (Lama guanicoe) en base a su dentición. Revista Argentina de Producción Animal 4(9): 965-983.

Payne, S. 1973. Kill-off patterns in sheep and goats: the mandibles from Asvan Kale. Anatolian Studies 23: 282-303.

Payne, S. 1987. References codes for wear stages in the mandibular cheek teeth of sheep and goats. Journal of Archaeological Science 14: 609-614.

Politis, G. G. 2002. South America: in the garden of forking paths, B. W. Cunliffe, W. Davies y C. Renfrew editores, Archaeology: the widening debate, pp. 193-244. University Press, Oxford.

Puig, S. y S. Monge. 1983. Determinación de la edad en Lama guanicoe (Muller). Deserta 7: 246-270.

Puig S, F. Videla y M. I. Cona. 1997. Diet and abundance of the guanaco (Lama guanicoe Muller 1776) in four habitats of northern Patagonia, Argentina. Journal of Arid Environments 36: 343-357.

R Core Team. 2016. R: A language and environment for statistical computing. R Foundation for Statistical Computing, Vienna, Austria. http://www.Rproject.org/.

Raedeke, K. 1979. "Population dynamics and sociology of the guanaco (Lama guanicoe) of Magallanes, Chile". Tesis Doctoral Inédita, Washington University, Seattle.

Reitz, E. J. y E. S. Wing. 1999. Zooarchaeology. Cambridge University Press, Cambridge.

Ruscillo, D. 2006. The table test: a simple technique for sexing canid humeri, D. Ruscillo editor, Recent advances in ageing and sexing animal bones, 62-67. BAR International Series British Archaeological Reports, Oxford.

Speth, J. 1983. Bison kills and bone counts: decision making by ancient hunters. University of Chicago Press, Chicago.

Spinage, C. A. 1973. A review of the age determination of mammals by means of teeth, with especial reference to Africa. East African Wildlife Journal 11: 165-187.

Stahl, P. 1999. Structural density of domesticated South American camelid skeletal elements and the archaeological investigation of prehistoric Andean charki. Journal of Archaeological Science 26: 1347-1368.

Steele, T. E. 2002. "Red deer: their ecology and how they were hunted by Late Pleistocene hominids in Western Europe". Tesis Doctoral Inédita. Stanford University, Stanford.

Stiner, M. 1990. The use of mortality patterns in archaeological studies of hominid predatory adaptations. Journal of Anthropological Archaeology 9: 305-351.

Wilson, B., C. Grigson y S. Payne. 1982. Ageing and Sexing Animal Bones from Archaeological Sites. BAR International Series British Archaeological Reports, Oxford. 